\title{
Multiple mating in the context of interspecific hybridization between two Tetramorium ant species
}

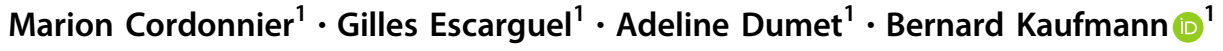

Received: 29 November 2019 / Revised: 29 February 2020 / Accepted: 11 March 2020 / Published online: 23 March 2020

(c) The Author(s), under exclusive licence to The Genetics Society 2020

\begin{abstract}
In eusocial Hymenoptera, haplodiploidy and polyandry may facilitate selection for hybridization. Interspecific hybridization is widespread in ants and can lead to hybrid inviability as well as the formation of new species through hybrid speciation. However, in ants, polyandry is uncommon. By analyzing microsatellite markers on 15 ant workers per colony, we show that the mating system of 28 pure colonies of Tetramorium immigrans, 15 pure colonies of Tetramorium caespitum, and 27 hybrid colonies is a monogyne/polyandrous mating system, with a higher mating rate in T. caespitum (mean $=2.4$ males vs. 1.7 in T. immigrans). Hybrid queens, but no hybrid fathers, were deduced from workers' genotypes, in accordance with Haldane's rule extended to haplodiploid organisms, which states that the haploid sex should more often be sterile or inviable. In five colonies, hybridization and multiple mating allowed the simultaneous production of both hybrid and nonhybrid offspring. Although rare, these situations hinted at asymmetrical, larger contributions of T. immigrans vs. T. caespitum males to offspring production. Together, these findings point toward a complex and dynamic mating system in $T$. immigrans and T. caespitum, and contribute to better understand interspecific hybridization mechanisms and their consequences on genetic and taxonomic diversity. The study of polyandry within a hybrid zone is unprecedented and opens new opportunities to better understand interspecific hybridization mechanisms and their short- to long-term consequences.
\end{abstract}

\section{Introduction}

Hybridization between species has been known for a long time and has received growing attention in the last few decades (e.g., Rhymer and Simberloff 1996; Allendorf et al. 2001), especially regarding its extent and effects across substantial fractions of biodiversity (Mallet 2008). Research on hybridization between species also provides unparalleled insights into the pre- and post-zygotic isolating mechanisms that drive speciation, and therefore raises questions relating to the mechanisms involved in interspecific mating systems.

Associate editor: Darren Obbard

Supplementary information The online version of this article (https:// doi.org/10.1038/s41437-020-0310-3) contains supplementary material, which is available to authorized users.

Marion Cordonnier

marion.cordonnier@hotmail.fr

1 Univ. Lyon, UMR5023 Ecologie des Hydrosystèmes Naturels et Anthropisés, ENTPE, CNRS, Université Lyon 1, Villeurbanne, F69622 Lyon, France
In the specific context of hybridization, mating behaviors (e.g., mate choice, species discrimination, and competition) influence whether species interbreed, and can therefore promote or impede behavioral reproductive isolation (Moore 1987; Parker and Partridge 1998; Irwin and Price 1999; Randler 2002; Lipshutz 2017). Bringing together hybridization and mating systems can thus offer important insights into their interaction and how this may impact the strength of barriers and asymmetries in gene flow (Pickup et al. 2019).

Interspecific hybridization is common and widespread in ants (Feldhaar et al. 2008), and has been already described in numerous genera, e.g., Solenopsis (Ross et al. 1987), Formica (Kulmuni et al. 2010; Purcell et al. 2016), Lasius (Van der Have et al. 2011), or Tetramorium (Steiner et al. 2010; Cordonnier et al. 2019a). Hybridization can lead to a wide diversity of results ranging from hybrid inviability or sterility to the formation of new species through hybrid speciation (Beresford et al. 2017). Combinations of mechanisms involved in reproductive behavior may facilitate selection for hybridization (Anderson et al. 2008), including haplodiploid sex determination systems and polyandry. On the one hand, the haplodiploid reproductive 
system, where haploid males from unfertilized eggs only inherit maternal genetic material, can mitigate outbreeding depression (i.e., reduction of fitness of hybrid offspring) compared with other organisms, as male fitness is preserved at the $\mathrm{F} 1$ generation because diploid queens that have mated with heterospecific haploid males still produce purebred sons via arrhenotokous parthenogenesis (Feldhaar et al. 2008; Kulmuni et al. 2010; Kronauer et al. 2011). When hybrids are fertile, truly hybrid males therefore are only produced in backcrossed colonies, by hybrid queens (Schilthuizen et al. 2011; Kronauer et al. 2011). Haploid males are particularly likely to show hybrid incompatibilities (Koevoets and Beukeboom 2009), and some ant species have therefore evolved elaborate mechanisms to avoid producing hybrid males, for instance transmission ratio distortion (i.e., whereby genetic incompatibilities cause allelic segregation to depart from the Mendelian ratio) depending on offspring sex (Kulmuni et al. 2010). On the other hand, eusocial Hymenoptera generally form colonies that contain a single reproductive queen and many functionally sterile diploid worker individuals. In ant species, gynes (virgin reproductive females) either mate only once (monandry) or several times (polyandry) (Heinze 2008). Nevertheless, polyandry is relatively uncommon in ants (reviewed in Strassmann 2001; Villesen et al. 2002; but see Hardy et al. 2008; Haapaniemi and Pamilo 2012). However, polyandry can increase genetic diversity in workers, leading to morphological, behavioral, or physiological variation among workers, which may raise colony fitness and provide benefits from heterosis in hybrid workers, resulting in positive selection for hybridization (e.g., Anderson et al. 2008). In eusocial insects, there obviously is a precopulatory mate-choice phase, but it involves a very restricted set of mate-quality cues (Boomsma 2013). In cases of interspecific hybridization combined with polyandry, considering mate choice becomes crucial. Being able to breed with a male of another species increases the probability of finding one or more partners, and could thus even prove reproductively advantageous (Rosenthal 2013). Nonacs (2006) pointed out that when population densities are low, females may not always be able to encounter conspecific males, and hybridization may be a "best of a bad situation" strategy to gain some reproductive success. Alternatively, a lack of mate preference and high levels of polyandry should result in the production of both F1 hybrid and nonhybrid offspring within the same colony (Anderson et al. 2008).

As in many species with internal fertilization, ant females possess a spermatheca, which is a specialized storage organ where sperm is stored between insemination and egg fertilization. Polyandry therefore results in the co-occurrence of different ejaculates in the female's reproductive tract (Jaffé et al. 2012), allowing sexual selection to operate after copulation. Mechanisms for postcopulatory sexual selection, which lead to biased paternity patterns, include sperm competition, where ejaculates from different males compete to fertilize eggs (Simmons 2001; but see Boomsma 2013) and cryptic female choice, where females influence which permanently stored sperm fertilizes their eggs (Eberhard 1996). In situations of hybridization between species, potentially negative consequences of hybridization can therefore be mitigated or even avoided when hybrid individuals mostly or exclusively become nonreproductive workers (Kronauer et al. 2011), or if fewer hybrid individuals are produced than purebred individuals, because of sexual selection processes, genetic caste determination (Nonacs 2006), or fitness decrease or increase (Umphrey 2006). In this last situation, selective processes unrelated to sexual selection can alter the viability or fitness of hybrid offspring.

The questions related to mate choice, sperm competition, and cryptic female choice in polyandrous eusocial Hymenoptera therefore often differ from those addressed in noneusocial mating systems, but their originality may allow experimental tests of the generality of sexual selection theory that cannot be performed in mating systems with remating promiscuity (Boomsma 2007). Although the processes involved are complex, such questions become even more exciting when considering systems involving interspecific hybridization. The present study investigates a situation resulting from such mechanisms in two monogynous Tetramorium cryptic species, namely $T$. immigrans Santschi 1927, and T. caespitum (Linnaeus 1758). Tetramorium immigrans and T. caespitum can hybridize (Wagner et al. 2017), and hybrids are fertile, leading to a high frequency of hybrid colonies in sympatric populations of these species (Cordonnier et al. 2019b).

In this paper, we analyze polyandry and asymmetrical contributions to offspring in the context of hybridization between species, by investigating mating systems in 28 pure colonies of $T$. immigrans, 15 pure colonies of $T$. caespitum, and 27 hybrid colonies. The main hypothesis tested is that some elements of the mating systems of these species, never depicted before, result in the hybridization pattern between species with a high frequency of hybrids and the existence of backcrosses demonstrated in previous studies, based on a study of the contribution to offspring, as well as the species or hybrid status of breeding males and females. The present study aimed to (1) test if both hybrid queens and males reproduce; (2) determine whether hybridization correlates to the polyandrous mating system of parental species, and if this pattern is modified along the latitudinal gradient; (3) provide an assessment of the bias in offspring production in hybrid colonies (e.g., capability of hybrids of both sexes to reproduce, production of both F1 hybrid and nonhybrid offspring within the same colony); (4) verify the hypothesis 
of random paternity allocation between species, i.e., the random contribution of males to the offspring, as opposed to mechanisms of postcopulatory sexual selection biasing worker paternity in colonies.

\section{Materials and methods}

The present study focuses on Tetramorium immigrans Santschi 1927 and T. caespitum (Linnaeus, 1758), two palearctic species of the $T$. caespitum complex largely distributed in western Europe and distinguishable using genetic (Cytochrome Oxidase I analysis, nuclear AFLP, and microsatellite markers) and morphometric characters on workers (Wagner et al. 2017; Cordonnier et al. 2019a).

\section{Microsatellite data}

Cordonnier et al. (2019a) collected workers of 544 colonies of T. immigrans, 698 colonies of $T$. caespitum, and 240 colonies of potential hybrids between $T$. immigrans and $T$. caespitum in southeastern France. For each colony, one worker was identified using nuclear DNA clustering based on a subset of workers identified using mtDNA cytochrome oxidase I sequencing. In the present study, we resampled 75 of these colonies: 30 colonies whose worker had a genotype associated with $T$. immigrans ( $Q$ value $>95 \%$ in Cordonnier et al. 2019a, values obtained using the software STRUCTURE v. 2.3.1; Pritchard et al. 2000), 15 whose worker had a genotype associated with $T$. caespitum ( $Q$ value $>95 \%$ in Cordonnier et al. 2019a), 15 potential F1 hybrids ( $Q$ value between 40 and $60 \%$ in Cordonnier et al. 2019a), and 15 potential backcrosses with $T$. immigrans (genotype associated between 60 and $80 \%$ to parental species in Cordonnier et al. 2019a). These colonies were randomly subsampled in 6 out of the 19 spatial areas described in Cordonnier et al. (2019a), corresponding to three latitudinally distinct spatial areas (Fig. 1) covering the whole 180$\mathrm{km}$ latitudinal extent of the hybrid zone described in Cordonnier et al. (2019b).

For 15 randomly selected workers per colony, DNA was extracted from whole individuals, which were crushed and then mixed with $150 \mu \mathrm{L}$ of Chelex ${ }^{\oplus} 100$ and $10 \mu \mathrm{L}$ of proteinase $\mathrm{K}\left(15 \mathrm{mg} \mathrm{mL}^{-1}\right)$ at room temperature; the solution was incubated at $55^{\circ} \mathrm{C}$ overnight (Casquet et al. 2012). For genotyping, 17 microsatellites developed for $T$. immigrans or T. tsushimae by Steiner et al. (2008) were organized in three multiplex PCR mixes. All three mixes had a total volume of $10 \mu \mathrm{L}$ with $1 \mathrm{X}$ MasterMix (kit type-it microsatellite PCR Qiagen $\left.n^{\circ} 206246\right)$ and $2 \mu \mathrm{L}$ of DNA. Mix 1 contained $0.08 \mu \mathrm{M}$ of tspE53a primers, and $0.2 \mu \mathrm{M}$ of tspE52b, tspE52d, tspE52k, ttsU55a, and ttsU56d primers. Mix 2 contained $0.15 \mu \mathrm{M}$ of tspE51oR3 primers, $0.2 \mu \mathrm{M}$ of
tspE51a, tspE51b, tspE51d, ttsU58i, and ttsU59j primers, and $0.3 \mu \mathrm{M}$ of tspE51i primers. Mix 3 contained $0.08 \mu \mathrm{M}$ of ttsU54e, $0.15 \mu \mathrm{M}$ of tspE52a, and $0.2 \mu \mathrm{M}$ of tspE53b and ttsU571 primers. Amplifications consisted of $5 \mathrm{~min}$ at $95^{\circ} \mathrm{C}$, then 32 cycles $\left(30 \mathrm{~s}\right.$ at $95^{\circ} \mathrm{C}, 90 \mathrm{~s}$ at $60^{\circ} \mathrm{C}$, and $30 \mathrm{~s}$ at $72^{\circ} \mathrm{C}$ ), and $30 \mathrm{~min}$ at $60^{\circ} \mathrm{C}$. All PCR products were analyzed with an ABI 3730xl sequencer (service provided by GENTYANE). Electropherograms were read and interpreted with Genemarker v.1.95 (SoftGenetics). One marker was discarded because it presented risks of misinterpretation at the reading stage. In addition, workers where alleles were not clearly legible for at least 12 markers were removed from the analysis, and colonies with less than 12 (pure T. immigrans or T. caespitum) or 14 (hybrid colonies) legible worker genotypes were discarded. Our final dataset included genotypes for 16 microsatellite markers (Tspe52d, Tspe52b, Tspe52k, Ttsu56d, Tspe53a, Ttsu55a, Ttsu59j, Tspe51i, Ttsu58i, Tspe51a, Tspe51o, Tspe51b, Tspe51d, Tspe52a, Tspe53b, and Ttsu571) for 28 putative T. immigrans colonies, 15 putative $T$. caespitum colonies, and 27 putative hybrid colonies (14 F1 and 13 backcrosses) with an average of 14.8 worker genotypes per colony, and a total of 1038 genotypes for 16 markers (basic statistics computed in GENALEX v.6; Peakall and Smouse 2006; Table S1).

\section{Observed number of matings}

Based on the identified worker genotypes, we first "manually" inferred the genotypes of queens and their mates in each colony to minimize potential errors. At each locus, two alleles shared by all the workers were assigned to the queen, while haploid fathers' genotypes were determined by the alleles unassigned to the mother. This pattern was then iterated over the 16 markers until reaching a minimal number of sires per queen. This allowed an individual correction of all potential genotyping errors to avoid overestimating the number of potential sires. Although time-consuming, such an approach minimizes errors compared with classically used software (e.g., COLONY, Matesoft) for which data monitoring and analysis are automated, quite often without post-process quality control. Here, scoring errors were checked and corrected one last time after identification of mothers and sires. Situations where more than one potential queen genotype was necessary to account for the data were not found in any of the 68 colonies tested. For each colony, the observed number of matings per queen (called observed paternity $K_{\text {obs }}$ in Jaffé (2014) and corresponding to the number of males siring offspring of a single queen estimated by genotyping worker offspring) was systematically recorded. Differences in the observed number of matings between species and areas were investigated by nonparametric Kruskal-Wallis tests coupled with Mann-Whitney-Wilcoxon tests for contrasting pairs (with a simple Bonferroni correction). 
Fig. 1 Map of the study area. Species ranges are indicated on the right, based on populations indicated by gray dots. The three sampled areas (North, Center, and South) are indicated as small dashed squares within the hybrid zone (large dotted square). 1: Tournus, 2: Mâcon, 3: Villefranche-sur-Saône, 4: Lyon, 5: Tournon sur Rhône, 6 : Valence. The main rivers are indicated in dark gray; altitude is indicated by grayscale (black $=$ high altitude).

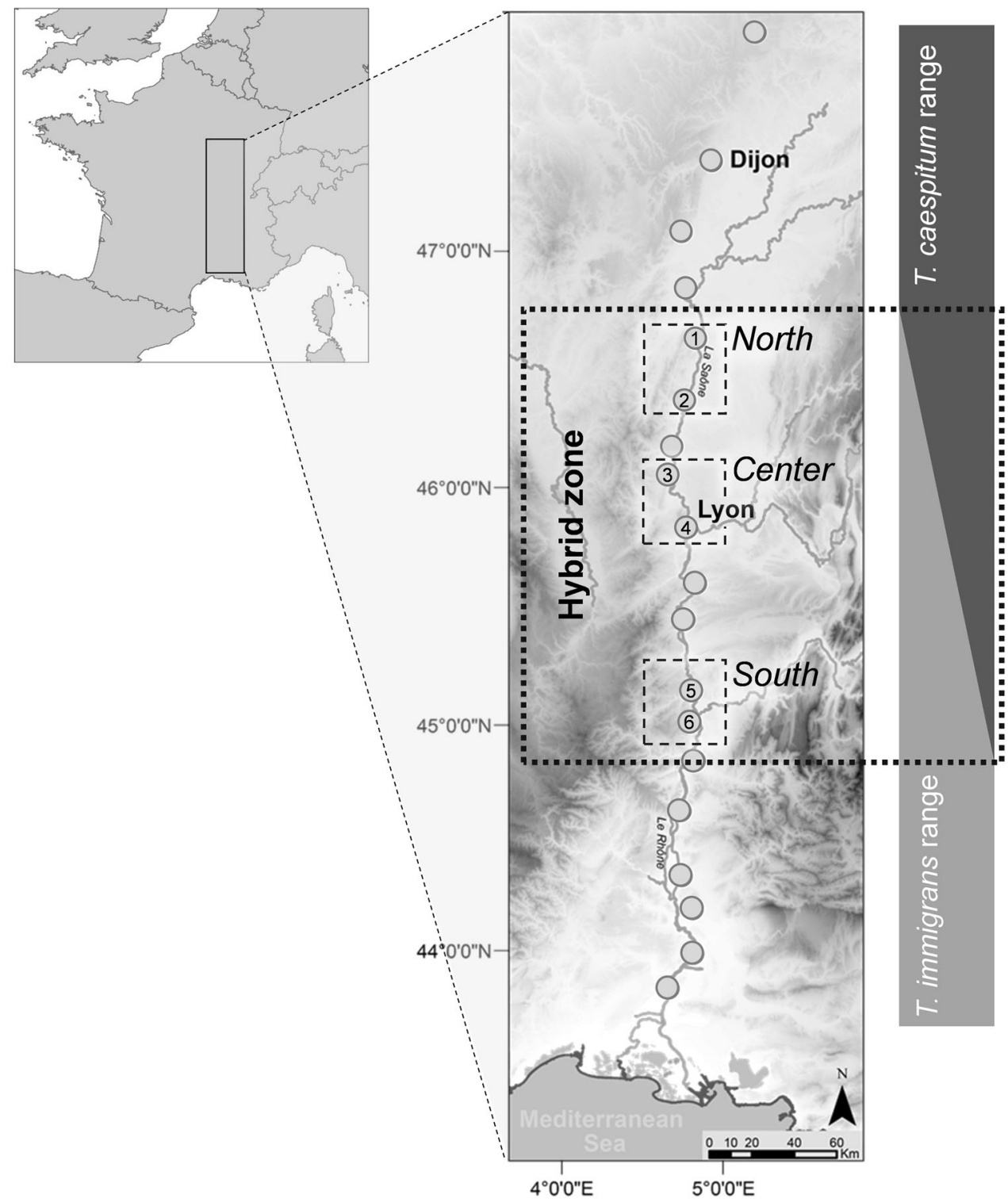

in period of 500,000 replicates. Clustering results were analyzed using CLUMPAK (Kopelman et al. 2015) based on a Markov clustering algorithm that identifies sets of highly similar runs grouped together in modes and separates these distinct groups of runs to generate a consensus solution for each distinct mode. Based on the consensus solution of the majority mode (no minority mode was found in this analysis), we obtained two distinct $Q$ values for each individual corresponding to their membership coefficient for each cluster (Fig. 2).

\section{Assignment of individuals}

Assignment of individuals was based on the four categories defined by NEWHYBRIDS (T. immigrans, T. caespitum, F1, and backcrossed hybrids), as this method has been 


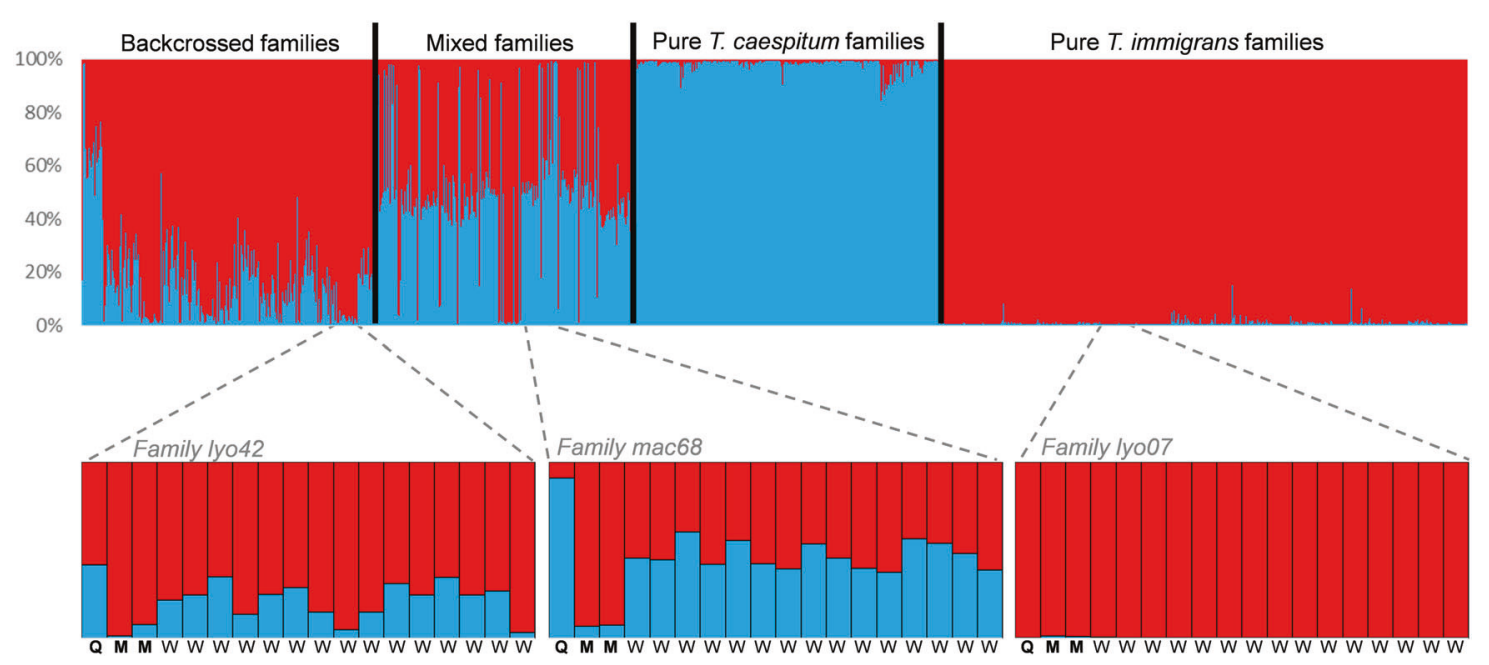

Fig. 2 Top-barplot obtained from STRUCTURE Bayesian clustering based on the 1008 worker genotypes and the 198 reconstructed genotypes of parents (68 females and 130 males). Each vertical line corresponds to an individual. Colors indicate membership to each cluster (T. immigrans in red, T. caespitum in blue). Bottom-

demonstrated to better discriminate hybrids in our system (Cordonnier et al. 2019b). To ensure a high accuracy of assignments and to exclude with certainty any misassignment to any of these potential categories, we applied a fivestep process:

(1) Individuals whose $Q$ values obtained from both STRUCTURE and NEWHYBRIDS value greater than 0.9 were assigned to a species (T. immigrans or T. caespitum).

(2) Individuals whose NEwHYBRIDS value in F1 or backcrossed hybrids was greater than 0.95 were assigned to the considered category (e.g., if the $\mathrm{NH}$ value in F1 category was 0.97 , the individual was assigned to F1 hybrids).

(3) Individuals whose $Q$ values corresponding to the T. immigrans group were comprised between 0.1 and 0.4, and whose NH-backcrossed hybrid values for T. immigrans were greater than 0.1 , were assigned to Backcrossed with T. immigrans (and reciprocally for T. caespitum). Individuals whose $Q$ values were comprised between 0.4 and 0.6 , and whose NH F1 values were greater than 0.1 , were assigned to the F1 hybrid.

(4) For all nonidentified workers, the assignment was deduced from full sister(s) identified in the offspring. For all nonidentified males, the assignment was deduced from the queen and accurately identified offspring. For instance, if all the offspring of a given male were accurately assigned to $F 1$ and the queen was accurately assigned to $T$. caespitum, a male whose $\mathrm{NH}$ value for $T$. immigrans $=1$ and $Q$ value $=0.88$ was assigned to $T$. immigrans, as no other explanation detailed barplots of three colonies representative from the different types of families (left: backcrossed, middle: mixed, and right: pure families), for workers (W), queens (Q), and males (M). The ID of the family is indicated in gray.

was compatible with, even if the $Q$ value is slightly under 0.9 .

(5) After these four steps, families were re-examined for consistency in order to confirm that previous assignments were accurate. In the case of incompatibility between worker offspring and parents identity (observed in 13 of the 70 colonies analyzed), we used both NH and $Q$ values obtained from STRUCTURE to better characterize these situations (see details in the "Results" section), except for two colonies discarded from the analysis because of discordance between assignment methods.

The final dataset is thus based on 1008 worker genotypes belonging to 68 colonies and the associated parental reconstructed genotypes.

\section{Results}

The inferred genotypes of queens showed that all colonies were monogynous, as a single maternal diploid genotype was consistent with the observed worker genotypes in the 68 colonies investigated. The inferred genotypes of males revealed that polyandry occurred in both species, with an overall mean of 1.912 observed mates for the 68 colonies studied. The five-step process of assignment detailed above allowed the identification of all 1008 analyzed workers, as well as the inferred 68 females and 130 males. In 11 colonies where worker offspring identities were incompatible with parents' identities and previous assignments, $\mathrm{NH}$ values and $Q$ values obtained by STRUCTURE were used to 
Table 1 Number of colonies in each sampling area and colony categories: pure $T$. immigrans or $T$. caespitum colonies containing only workers, queen, and male(s) of pure parental species, mixed colonies containing pure and hybrid workers but only pure parents, and backcrossed colonies containing hybrid workers and parents.

\begin{tabular}{llll}
\hline & North & Center & South \\
\hline T. immigrans colonies & 9 & 9 & 9 \\
T. caespitum colonies & 5 & 6 & 4 \\
Mixed colonies & 4 & 3 & 4 \\
Backcrossed colonies & 5 & 6 & 4 \\
\hline
\end{tabular}

better understand these situations. These mismatches corresponded to either (1) an overdetection of individuals belonging to parental species in situations where a hybrid queen strongly backcrossed with $T$. immigrans mated with pure $T$. immigrans males (observed in 1 of the 11 situations), or (2) an overdetection of F1 hybrids in situations where a hybrid queen strongly backcrossed with $T$. immigrans mated with pure $T$. caespitum males (1/11 situations). Overall, four categories of colonies were identified: (1) 27 pure $T$. immigrans colonies containing only $T$. immigrans workers, queen, and male(s); (2) 15 pure T. caespitum colonies containing only $T$. caespitum workers, queen, and male(s); (3) 11 mixed colonies containing pure and hybrid workers but only pure parents (queen or males); (4) 15 backcrossed colonies containing hybrid workers and parents. The numbers of colonies in each category and each sampling area are detailed in Table 1.

Among the 15 backcrossed colonies, 6 corresponded to an F1 female mated with pure males and 9 involved a backcrossed female mated with pure males (Fig. 3). Remarkably, none of the 25 males involved in these 15 backcrossed colonies were hybrids.

The observed number of matings was significantly higher in $T$. caespitum $\left(K_{\mathrm{obs}}=2.400 \pm 0.1309\right)$ than in $T$. immigrans $\left(K_{\mathrm{obs}}=1.679 \pm 0.1156\right.$; Mann-Whitney-Wilcoxon $W=328.5, p$ value $=0.0008)$ (Fig. 4). Hybrid colonies had an intermediate observed number of matings $\left(K_{\mathrm{obs}}=\right.$ $1.955 \pm 0.0753$ ) compared with parental species. Differences of the observed number of matings between areas within each species suggested that polyandry differed latitudinally between areas (T. immigrans: $\mathrm{K}-\mathrm{W}$ chi-squared $=$ $5.65, \mathrm{df}=2, p$ value $=0.059 ; T$. caespitum: $\mathrm{K}-\mathrm{W}$ chisquared $=5.25, \mathrm{df}=2, p$ value $=0.072$ ) with nearly significantly higher level of polyandry in the south compared with the north in T. immigrans (Mann-Whitney-Wilcoxon $W=16.5, p$ value $=0.055)$ (Fig. 4).

In the 11 mixed colonies (i.e., colonies involving heterospecific mating), the mean number of mates detected was $2( \pm 0.6325)$. Five colonies were characterized by the simultaneous presence of both conspecific and heterospecific males (four situations involving a $T$. caespitum queen, and

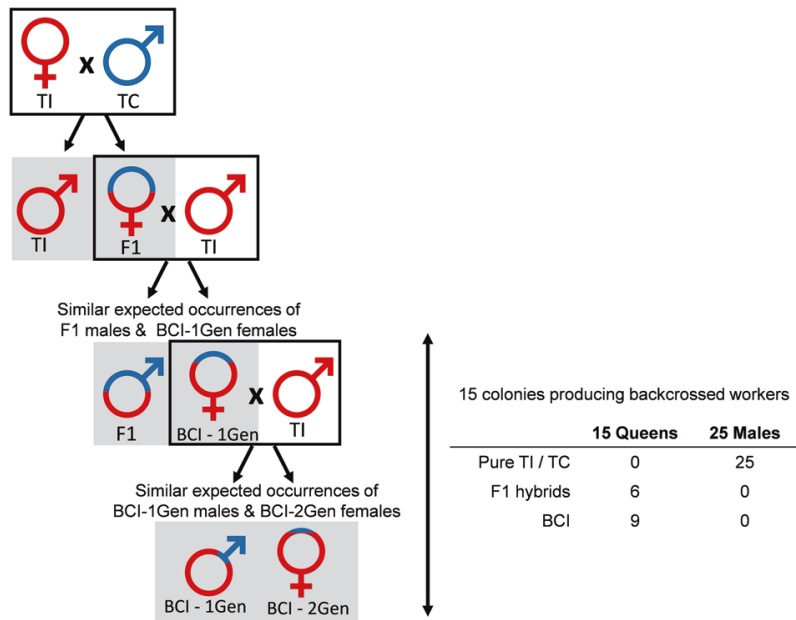

Fig. 3 Illustration of the situations observed in the 15 backcrossed colonies. Colors indicate the proportion of genotype associated with one or the other species. TI T. immigrans. TC T. caespitum. F1 F1 hybrid. BCI-1 Gen backcross with T. immigrans (first generation), BCI-2 Gen backcross with T. immigrans (second generation). Gray squares indicate the offspring produced and dark squares the mating pattern generating the next generation.

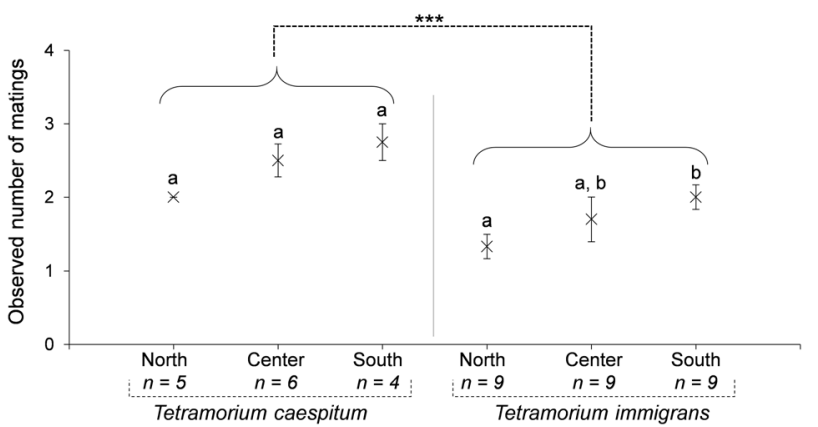

Fig. 4 Observed number of mating $\left(K_{\text {obs }}\right)$ in each pure species (T. caespitum and T. immigrans) and for each area (North, Center, and South) in the hybrid zone. Stars indicates a significant difference $(p<0.001)$ between species assessed by Mann-Whitney-Wilcoxon test. Letters indicate significant differences $(p<0.05)$ between areas assessed by Mann-Whitney-Wilcoxon test with a simple Bonferroni correction.

one situation involving a $T$. immigrans queen, mean number of mates detected $=2.4 \pm 0.5477$ ). In four situations, worker offspring were overwhelmingly sired by $T$. immigrans males (Fig. 5).

\section{Discussion}

\section{Mating system}

By investigating the mating system of 27 pure colonies of Tetramorium immigrans, 15 pure colonies of Tetramorium caespitum, and 26 hybrid colonies, we demonstrated that 


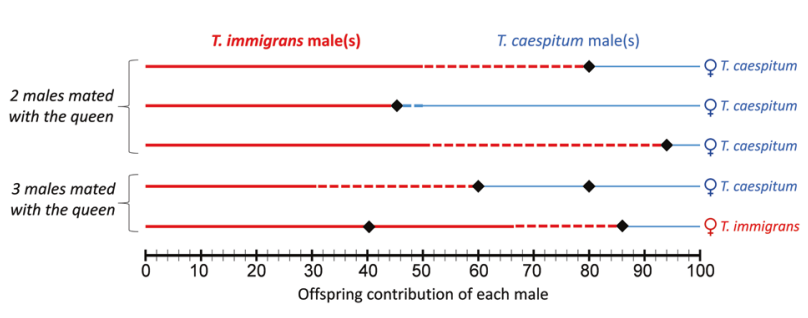

Fig. 5 Offspring contribution of each male in the five mixed colonies where females mated with both conspecific and heterospecific males. The diamonds separate the contributions of the different males. Red lines correspond to $T$. immigrans males' contributions and blue lines correspond to T. caespitum males' contributions. Dashed lines indicate the excess of offspring compared with random expectation (e.g., $50 \%$ offspring per male when two males are mated). The species of the queen is indicated on the right.

both $T$. immigrans and $T$. caespitum displayed a monogyne/ polyandrous mating system, with a higher mating rate in T. caespitum. To our knowledge, our study is the first to describe polyandry in these two species. The occurrence of multiple mating has raised theoretical and experimental attention to issues related to sexual selection and sexual conflict, such as the mechanisms involved in sperm competition and cryptic female choice, as well as the costs and benefits that females may incur with multiple mating (Pizzari and Wedell 2013). In social Hymenoptera (ants, bees, and wasps), the adaptive significance of polyandry is often explained by hypotheses suggesting that polyandry is advantageous to queens because the resultant increase in genetic variation within colonies leads to increased colony performance (Keller and Reeve 1994; but see Fjerdingstad et al. 2003 or Fournier et al. 2008). These hypotheses are also often put forward to explain the value of hybrid offspring, as increased allelic variation and transfer of adaptively important genetic variation may increase the fitness of the introgressed lineage (Twyford and Ennos 2012). Future measurements of colony performance in the species studied here (and their hybrids) as a function of their degree of polyandry should be considered; as such, a multiple mating and hybridization system offers unique insight into important eco-evolutionary mechanisms such as sexual selection and sexual conflict or species interaction and niche partitioning. Hypotheses for multiple mating also include a need for more sperm than provided by a single male (Crozier and Fjerdingstad 2001), especially regarding the sperm limitation hypothesis suggesting that queens are able to produce larger or longer-lived colonies if they obtain more sperm than delivered by a single male (Cole 1983). Following such hypothesis, the lower number of mates found in $T$. immigrans colonies could be explained by the fact that $T$. immigrans males produce more sperm than $T$. caespitum males, therefore implying that fewer $T$. immigrans males would be needed to fill a gyne's spermatheca.
In the case of hybridization between species, polyandry may lead females to mate with both con- and heterospecific males, and to produce varying proportions of hybrid offspring. From a purely statistical view, polyandry should spread hybridization faster than monandry, because if females would randomly mate with males of both species, more mates should lead to an increased probability of hybrid offspring, independent of the female's species. For instance, in a theoretical situation with an initial population mixing $50 \%$ of each parental species, the random mating of females with one, two, or three potential males systematically increases the probabilities of producing hybrid offspring, with probabilities of producing hybrid offspring of $0.5,0.75$, and 0.875 with one, two, or three mates, respectively (Fig. S1). The coexistence of hybridization and multiple mating observed in the system described here should then accelerate the spread of introgression.

\section{Latitudinal variation of mating frequency}

In this study, the level of polyandry seemed to increase at lower latitudes although data are too scarce to confirm a latitudinal pattern. Our observations are consistent with Corley and Fjerdingstad (2011), who investigated two populations of Lasius niger in North Europe vs. two populations in South Europe, and concluded that multiple mating by queens was far more frequent in the two southern populations than in the two northern populations. These authors suggested that this pattern arose because multiple mating might increase fitness for queens and colonies in Southern European climates, whereas only exceptionally good colonies could survive in harsher northern environments, thus favoring single vs. multiple paternity that could reduce variance in colony performance. As far as the two Tetramorium species studied here are concerned, it should be interesting to extend the present study to a broader latitudinal range, and to investigate situations outside the hybrid zone, in which other mechanisms might control reproductive mechanisms within and between these species, such as intraspecific competition (Umphrey 2006) or differences in habitats or microhabitats of these two species (Cordonnier et al. 2019c).

\section{Hybrid females, but not males, produce offspring}

Our results clearly showed the reproductive capability of hybrid females as both F1 and backcrossed queens have been found. However, no hybrid male was inferred as a contributor to the paternity of workers. The fact that potential mating combinations resulting in unviable offspring were not detected does not necessarily involve that hybrid males do not exist, but that they do not produce viable offspring, or not enough to be detected in this study. 
Introgression into females but not into males is compatible with the extended Haldane's rule for haplodiploids, which states that the haploid sex should more often be sterile or inviable (Koevoets and Beukeboom 2009). Nevertheless, to date, this pattern has been rarely documented in ants. The only example in the literature involved hybrids between the red wood ants Formica aquilonia and $F$. polyctena (Kulmuni et al. 2010). In this system, two genetic groups coexist in highly polygynous nests; the alleles of one genetic group are found in the diploid individuals (i.e., females) but not the haploid males of the other genetic group, leading to hybrid females (workers and queens) but not to hybrid males (Kulmuni et al. 2010). Kulmuni and Pamilo (2014) tested two hypotheses to explain this pattern: the selection and segregation hypotheses. On the one hand, the selection hypothesis implies a strong post-zygotic selection against hybrid males, because recessive incompatibilities can be masked in diploid heterozygous females but not in haploid males. In this case, hybrid males die but females survive. On the other hand, the segregation hypothesis states that hybridization has led to the formation of two independently segregating sets of alleles, one always transmitted from the queens to their sons ( $Y$ type) and the other to their daughters (X type) after fertilization by sperm carrying the paternal complement (Y), leading to hybrid females but only pure males. According to Lowry and Willis (2010), this second situation could contribute to hybrid incompatibilities and lead to reproductive isolation of hybrids. In this context, it should be relevant to test this hypothesis in the present system, e.g., by monitoring genotypes at various stages of development (very young embryos, sexual larvae and pupae, worker larvae and pupae, adult females, males, and workers), as done by Kulmuni and Pamilo (2014).

\section{Asymmetrical contributions to offspring of T. immigrans vs. T. caespitum males}

A major feature of the present study is the discovery of the coexistence of hybridization and multiple mating, allowing the simultaneous production of both hybrid and nonhybrid offspring within a single colony. Nevertheless, the production of F1 hybrid and nonhybrid offspring within the same colony was rare (only five colonies) and did not allow conclusions to be drawn on the hypothesis of a random paternity allocation between species. The results did not suggest higher mating frequencies in colonies whose queens were mated with both conspecific and heterospecific males than in the pure-mated groups, suggesting that multiple mating was not a type of insurance against a poor initial mating. However, our study provided a preliminary assessment of the variation of offspring production in hybrid colonies, and suggested asymmetrical contributions to offspring of $T$. immigrans vs. T. caespitum males (although this could not be statistically tested). Although causality cannot be established directly from the patterns of paternity biases observed here, these patterns raise questions worthy of investigation, particularly with respect to the potential roles of postcopulatory sexual selection and sexual conflict in social evolution (Jaffé et al. 2012). As paternity skew across worker cohorts in the same colony seemed to occur, it would be worth checking whether this might simply indicate a difference in ejaculate volumes between species (more sperm produced or injected by $T$. immigrans males, as suggested above), or if such asymmetrical contributions are due to a difference in worker survival rate depending on the environment of the nest, or reflect differential larval growth rather than temporary variable sperm use owing to incomplete sperm mixing (FernándezEscudero et al. 2002; Boomsma 2013).

Together, our findings paint the picture of a complex and dynamic mating system in Tetramorium immigrans and T. caespitum. Numerous studies have already focused on hybridization, and even more on multiple mating, but so far, few if any have described a system combining both polyandry and interspecific hybridization, making it worth investigating for several reasons. One reason is that, as far as we know, the study of polyandry within a hybrid zone is totally unprecedented. It could also be an opportunity to investigate sexual selection processes in social Hymenoptera and an ideal system to test the numerous hypotheses about selective processes involved in the evolution of multiple mating. Finally, further inquiring into the hybrid Tetramorium situation should contribute to better understand interspecific hybridization mechanisms and their short- to long-term consequences on genetic and taxonomic diversity in a world facing human-induced global changes.

\section{Data availability}

Data are available from the Dryad Digital Repository: https:// datadryad.org/stash/dataset/doi:10.5061/dryad.qjq2bvqcb.

Acknowledgements This study was funded by the Conseil Départemental de l'Isère. It was also supported by the French National Research Agency (ANR) through the LABEX IMU (ANR-10-LABX0088) of Université de Lyon, within the program "Investissements d'Avenir" (ANR-11-IDEX-0007). We also thank temporary lab assistants, E. Vernay, M. Reina, L. Herpe, M. Dumet, and C. Richalet, for their invaluable help.

\section{Compliance with ethical standards}

Conflict of interest The authors declare that they have no conflict of interest.

Publisher's note Springer Nature remains neutral with regard to jurisdictional claims in published maps and institutional affiliations. 


\section{References}

Allendorf FW, Leary RF, Spruell P, Wenburg JK (2001) The problems with hybrids: setting conservation guidelines. Trends Ecol Evol 16:613-622

Anderson EC, Thompson EA (2002) A model-based method for identifying species hybrids using multilocus genetic data. Genetics 160:1217-1229

Anderson KE, Novak SJ, Smith JF (2008) Populations composed entirely of hybrid colonies: bidirectional hybridization and polyandry in harvester ants. Biol J Linn Soc 95:320-336

Beresford J, Elias M, Pluckrose L, Sundström L, Butlin RK, Pamilo P, Kulmuni J (2017) Widespread hybridization within moundbuilding wood ants in Southern Finland results in cytonuclear mismatches and potential for sex-specific hybrid breakdown. Mol Ecol 26:4013-4026

Boomsma JJ (2007) Kin selection versus sexual selection: why the ends do not meet. Curr Biol 17:R673-R683

Boomsma JJ (2013) Beyond promiscuity: mate-choice commitments in social breeding. Philos Trans R Soc Lond B Biol Sci 368:20120050

Casquet J, Thebaud C, Gillespie RG (2012) Chelex without boiling, a rapid and easy technique to obtain stable amplifiable DNA from small amounts of ethanol-stored spiders. Mol Ecol Resour 12:136-141

Cole BJ (1983) Multiple mating and the evolution of social behavior in the Hymenoptera. Behav Ecol Sociobiol 12:191-201

Cordonnier M, Gayet T, Escarguel G, Kaufmann B (2019b) From hybridization to introgression between two closely related sympatric ant species. J Zool Syst Evol Res 57:778-788

Cordonnier M, Gibert C, Bellec A, Kaufmann B, Escarguel G (2019c) Multi-scale impacts of urbanization on species distribution within the genus Tetramorium. Land Ecol 34:1937-1948

Cordonnier M, Bellec A, Dumet A, Escarguel G, Kaufmann B (2019a) Range limits in sympatric cryptic species: a case study in Tetramorium pavement ants (Hymenoptera: Formicidae) across a biogeographical boundary. Insect Conserv Divers 12:109-120

Corley M, Fjerdingstad EJ (2011) Mating strategies of queens in Lasius niger ants-is environment type important? Behav Ecol Sociobiol 65:889-897

Crozier RH, Fjerdingstad EJ (2001) Polyandry in social Hymenoptera - disunity in diversity? In: Annales Zoologici Fennici, Finnish Zoological and Botanical Publishing Board. pp. 267-285

Eberhard W (1996) Female control: sexual selection by cryptic female choice. Princeton University Press: New Jersey

Feldhaar H, Foitzik S, Heinze J (2008) Lifelong commitment to the wrong partner: hybridization in ants. Philos Trans R Soc Lond B Biol Sci 363:2891-2899

Fernández-Escudero I, Pamilo P, Seppä P (2002) Biased sperm use by polyandrous queens of the ant Proformica longiseta. Behav Ecol Sociobiol 51:207-213

Fjerdingstad EJ, Gertsch PJ, Keller L (2003) The relationship between multiple mating by queens, within-colony genetic variability and fitness in the ant Lasius niger. J Evol Biol 16:844-853

Fournier D, Battaille G, Timmermans I, Aron S (2008) Genetic diversity, worker size polymorphism and division of labour in the polyandrous ant Cataglyphis cursor. Anim Behav 75:151-158

Haapaniemi K, Pamilo P (2012) Reproductive conflicts in polyandrous and polygynous ant Formica sanguinea. Mol Ecol 21:421-430

Hardy OJ, Pearcy M, Aron S (2008) Small-scale spatial genetic structure in an ant species with sex-biased dispersal. Biol J Linn Soc 93:465-473

Van der Have TM, Pedersen JS, Boomsma JJ (2011) Mating, hybridisation and introgression in Lasius ants (Hymenoptera: Formicidae). Myrmecol News 15:109-115
Heinze $\mathbf{J}$ (2008) The demise of the standard ant (Hymenoptera: Formicidae). Myrmecol News 11:9-20

Irwin DE, Price T (1999) Sexual imprinting, learning and speciation. Heredity $82: 347$

Jaffé R (2014) An updated guide to study polyandry in social insects. Sociobiology $61: 1-8$

Jaffé R, Garcia-Gonzalez F, den Boer S, Simmons LW, Baer B (2012) Patterns of paternity skew among polyandrous social insects: what can they tell us about the potential for sexual selection? Evolution 66:3778-3788

Keller L, Reeve HK (1994) Genetic variability, queen number, and polyandry in social Hymenoptera. Evolution 48:694-704

Koevoets T, Beukeboom LW (2009) Genetics of postzygotic isolation and Haldane's Rule in haplodiploids. Heredity 102:16-23

Kopelman NM, Mayzel J, Jakobsson M, Rosenberg NA, Mayrose I (2015) Clumpak: a program for identifying clustering modes and packaging population structure inferences across K. Mol ecol resour 15:1179-1191

Kronauer DJ, Johnson RA, Boomsma JJ (2007) The evolution of multiple mating in army ants. Evolution 61:413-422

Kronauer DJ, Peters MK, Schöning C, Boomsma JJ (2011) Hybridization in East African swarm-raiding army ants. Front Zool 8:20

Kulmuni J, Pamilo P (2014) Introgression in hybrid ants is favored in females but selected against in males. Proc Natl Acad Sci USA 111:12805-12810

Kulmuni J, Seifert B, Pamilo P (2010) Segregation distortion causes large-scale differences between male and female genomes in hybrid ants. Proc Natl Acad Sci USA 107:7371-7376

Lipshutz SE (2017) Interspecific competition, hybridization, and reproductive isolation in secondary contact: missing perspectives on males and females. Curr Zool 64:75-88

Lowry DB, Willis JH (2010) A widespread chromosomal inversion polymorphism contributes to a major life-history transition, local adaptation, and reproductive isolation. PLoS Biol 8:e1000500

Mallet J (2008) Hybridization, ecological races and the nature of species: empirical evidence for the ease of speciation. Philos Trans R Soc Lond B Biol Sci 363:2971-2986

Moore WS (1987) Random mating in the Northern Flicker hybrid zone: implications for the evolution of bright and contrasting plumage patterns in birds. Evolution 41:539-546

Nonacs P (2006) Interspecific hybridization in ants: at the intersection of ecology, evolution, and behavior. Ecology 87:2143-2147

Parker GA, Partridge L (1998) Sexual conflict and speciation. Philos Trans R Soc Lond B Biol Sci 353:261-274

Peakall ROD, Smouse PE (2006) GENALEX 6: genetic analysis in Excel. Population genetic software for teaching and research Mol ecol 6:288-295

Pickup M, Brandvain Y, Fraisse C, Yakimowski S, Barton NH, Dixit $\mathrm{T}$ et al. (2019) Mating system variation in hybrid zones: facilitation, barriers and asymmetries to gene flow. New Phytologist 224:1035-1047

Pizzari T, Wedell N (2013) The polyandry revolution. Philos Trans R Soc Lond B Biol Sci 368:20120041

Pritchard JK, Stephens M, Donnelly P (2000) Inference of population structure using multilocus genotype data. Genetics 155:945-959

Purcell J, Zahnd S, Athanasiades A, Türler R, Chapuisat M, Brelsford A (2016) Ants exhibit asymmetric hybridization in a mosaic hybrid zone. Mol ecol 25:4866-4874

Randler C (2002) Avian hybridization, mixed pairing and female choice. Anim Behav 63:103-119

Rhymer JM, Simberloff D (1996) Extinction by hybridization and introgression. Annu Rev Ecol Evol Syst 27:83-109

Rosenthal GG (2013) Individual mating decisions and hybridization. J Evol Biol 26:252-255 
Ross KG, Meer RKV, Fletcher DJ, Vargo EL (1987) Biochemical phenotypic and genetic studies of two introduced fire ants and their hybrid (Hymenoptera: Formicidae). Evolution 41: 280-293

Schilthuizen M, Giesbers MCWG, Beukeboom LW (2011) Haldane's rule in the 21 st century. Heredity 107:95

Simmons LW (2001) Sperm competition and its evolutionary consequences in the insects (Vol. 68), Princeton University Press: New Jersey

Steiner FM, Seifert B, Moder K, Schlick-Steiner BC (2010) A multisource solution for a complex problem in biodiversity research: description of the cryptic ant species Tetramorium alpestre sp. n. (Hymenoptera: Formicidae). Zool Anz 249:223-254

Steiner FM, Arthofer W, Schlick-Steiner BC, Crozier RH, Stauffer C (2008) Twenty four new microsatellite markers in two invasive pavement ants, Tetramorium sp. E and T. tsushimae (Hymenoptera: Formicidae). Conserv genet 9:757-759

Strassmann J (2001) The rarity of multiple mating by females in the social Hymenoptera. Insectes soc 48:1-13

Twyford AD, Ennos RA (2012) Next-generation hybridization and introgression. Heredity 108:179

Umphrey GJ (2006) Sperm parasitism in ants: selection for interspecific mating and hybridization. Ecology 87:2148-2159

Villesen P, Murakami T, Schultz TR (2002) Identifying the transition between single and multiple mating of queens in fungus-growing ants. Proc R Soc B 269:1541-1548

Wagner HC, Arthofer W, Seifert B, Muster C, Steiner FM, SchlickSteiner BC (2017) Light at the end of the tunnel: Integrative taxonomy delimits cryptic species in the Tetramorium caespitum complex (Hymenoptera: Formicidae). Myrmecol News 25:95-129 